\title{
Organotypic Cultures of HepG2 Cells for In Vitro Toxicity Studies
} Daniel Mueller, Anika Koetemann and Fozia Noor*

Biochemical Engineering, Geb. A1 5, Saarland University, D-66123 Saarbruecken, Germany

\begin{abstract}
Tissue engineering of human liver cells in a three dimensional cell culture system could improve pharmacological studies in terms of drug metabolism, drug toxicity or adverse drug effects by mimicking the in vivo situation. In this study, we produced 3D organotypic cultures of HepG2 cells using the hanging drop method. 250 - 8000 seeded cells formed organotypic cultures within 2-3 days which increased in size during the first week. Viability and metabolic parameters (glucose, lactate) were analyzed during almost three weeks of cultivation. Liver specific albumin production was higher in the organotypic cultures as compared to both monolayer and collagen-sandwich cultures. Amino acid quantification revealed high production of glutamate as well as uptake of glutamine, alanine and branched-chain amino acids. CYP1A induction capacity was significantly improved by organotypic cultivation. The acute toxicity (24 h) of tamoxifen, an anti-cancer drug, was lower in the 3D cultures as compared to monolayer and collagen-sandwich cultures. This could be explained by a higher drug efflux through membrane transporter (MRP-2). We conclude that the engineered HepG2 cultures could be used for the investigation of CYP450 induction, anti-cancer drug effects and for the study of chemotherapy resistance. Applied to other cell types such as the human primary cells these 3D organotypic cultures may have potential in long term toxicity screening of compounds.
\end{abstract}

Keywords: Tissue engineering; Organotypic cultures; Spheroids; Liver cells; Drug metabolism; Toxicity; Metabolite profiling

\section{Introduction}

The field of tissue engineering has advanced tremendously in recent years. In preclinical drug development the challenge remains the design and manufacturing of cellular structures which mimic the in vivo situation. In a tissue, cell-cell interactions as well as cell contacts to the extracellular matrix and extracellular factors like signal molecules are promoted. The interactions between the cells and the environment strongly influence the behavior and functionality of the whole tissue. Focal adhesions anchor the cytoskeleton to the plasma membrane and transmit extracellular signals from the matrix into the cell $[1,2]$. In experimental 2D culture, this complex network is lost and an artificial cellular architecture is built up. Moreover, an unnatural polarity is formed since the upper cell side contacts the culture medium and the lower is attached to cell culture dish surfaces, resulting in a polar distribution of adhesion factors [3].

Different 3D cell culture techniques show the improvement of function, differentiation and viability as compared to conventional 2D cultures [4]. Biodegradable polymers such as poly(glycolic acid), poly(lactic acid) or their copolymer poly (lactic-co-glycolic acid) were successfully used to enhance cellular function [5] and were applied to tissue engineering [6]. Alternatively, hydrogels, a network of hydrophilic polymer chains dispersed in water, were used to set up a 3D environment for various cell types such as liver cells, bone and cartilage [7-10]. Alginate microencapsulation of hepatocytes was recently shown to enhance long-term cultivation [11] and continuous perfusion of these aggregates further improves liver-specific functions [12]. Matrigel-based systems are another alternative for 3D cultivation. Containing ingredients of the extracellular matrix such as collagen, laminin, fibronectin or elastin, they can at least partly replace its functions [13]. Although these gel-based systems are useful tools to facilitate a 3D environment, gel preparation, storage, batch to batch differences and insufficient chemical composition for matrigels and xenogenic origin compromises their application.

Complex systems such as $3 \mathrm{D}$ bioreactors were developed as extracorporeal liver support [14-17]. These systems were down-scaled for experimental purposes and it was shown that primary hepatocytes form 3D tissue-like structures within the bioreactors and could be kept viable and functional for three weeks [18]. Moreover, the cultivation of spheroids in stirred bioreactors also improves liver-specific functionality compared to monolayers [19]. Again these systems require high technical expertise and are not always reproducible.

As a further 3D cell culture approach, multi-cellular spheroids represent a promising tool in tissue engineering since it was shown that cellular reorganization and 3D architecture in such spheroids better reflect in vivo situation but also mimic solid tumors in case of oncological studies [20]. Liver cells are of main interest for the investigation of drug-induced effects and drug metabolism and the improvement of in vitro test methods for hepatotoxicity is still needed [21]. The HepG2 cell line was successfully applied for the investigation of the effects of drugs in subtoxic concentrations in terms of respiration and cell metabolism [22-24]. Moreover, when grown in 3D systems, HepG2 cells showed enhanced viability and functionality [25-28]. In spheroid cultures, it was observed that HepG2 cells have higher drug efflux activity compared to monolayers [29]. Multi drug resistance (MDR) is shown by tumor cells and leads to a high drug efflux via transporters such as MDR-1 or MRP.

In general, the HepG2 cell line has the advantages of reproducibility, easy handling and availability, but is limited in its drug metabolism capacities. For example, CYP3A4 as the most important phase I enzyme is not expressed in HepG2 cells and only CYP1A1 and CYP2D6

*Corresponding author: Fozia Noor, Biochemical Engineering Institute, Saarland University, Campus A 1 5, D-66123 Saarbruecken, Germany, Tel: +496813022205; Fax: +496813024572; E-mail: fozia.noor@mx.uni-saarland.de

Received November 01, 2011; Accepted December 08, 2011; Published December 09, 2011

Citation: Mueller D, Koetemann A, Noor F (2011) Organotypic Cultures of Hepg2 Cells for In Vitro Toxicity Studies. J Bioengineer \& Biomedical Sci S2:002. doi:10.4172/2155-9538.S2-002

Copyright: ( 2011 Mueller D. This is an open-access article distributed under the terms of the Creative Commons Attribution License, which permits unrestricted use, distribution, and reproduction in any medium, provided the original author and source are credited. 
shows expression rates approximately in the range of primary human hepatocytes [30]. Recently, it was proposed that engineering the cell culture environment for a better reflection of the in vivo situation would improve drug development at an early stage [31]. HepG2 spheroid cultures therefore seem to be promising as in vitro assay system, at least for acute and parent compound toxicity screening.

In this study, we used a commercially available 96 well plate system for the generation of 3D spheroids using the hanging drop method. We investigated the HepG2 spheroid formation over time including growth in size. We cultivated the spheroids in low-serum conditions for more than two weeks and assessed cell viability, albumin production and metabolism in the 3D cultures. Moreover, we investigated drug metabolism capacities by CYP1A induction and tested the effects of tamoxifen on the 3D HepG2 spheroids. Tamoxifen is a non-steroidal selective estrogen-receptor (ER) modulator applied in the therapy of ER-positive breast cancer. Tamoxifen is mainly metabolized by CYP3A4 and CYP2D6 to active metabolites [32]. However, it was reported that tamoxifen is cytotoxic in non-metabolizing cell lines, implicating parent compound toxicity [33]. We tested tamoxifen on the HepG2 organotypic cultures and compared the results with those obtained with 2D monolayer and collagen-sandwich cultures. We also investigated the activity of MRP-2 transporters. Our results demonstrate the potential of engineered HepG2 spheroids as in vitro model to screen anti-cancer drugs and their effects on cancer tissue as well as for the investigation of MDR activity in chemotherapy resistance. In addition, such organotypic cultures of other cells (including co-cultures) such as primary human cells (e.g. liver) can also be used to develop micro-tissues which may be used in long term toxicological screening of compounds.

\section{Material and methods}

\section{Cell culture}

The human hepatoblastoma cell line (HepG2) was obtained from the German collection of microorganisms and cell cultures (DMSZ, Braunschweig, Germany). Cells were maintained in Williams Medium E supplemented with penicillin/streptomycin $(100 \mathrm{U} / 100 \mu \mathrm{g} / \mathrm{ml})$ and $10 \%$ FCS. The cells were kept at $37^{\circ} \mathrm{C}$ in a cell incubator (Memmert $\mathrm{GmbH}$, Schwabach, Germany) at $95 \%$ relative humidity with $5 \% \mathrm{CO}_{2}$ supply. Viability was assessed by the trypan blue exclusion method. Cells were counted using an automated cell counter (Countess, Invitrogen, Karlsruhe, Germany).

\section{Monolayer / Collagen-Sandwich}

For monolayer culture (ML), cells were seeded in conventional 96-multiwell plates. For collagen-sandwich cultures (CS), the plates were coated with collagen I solution $(0.75 \mathrm{mg} / \mathrm{ml})$ for $1 \mathrm{~h}$ at $37^{\circ} \mathrm{C}$ before being seeded. After 4 hours, medium was aspirated and the second collagen layer was added as before. In both cultures, $5^{*} 10^{4}$ cells / well were seeded.

\section{Organotypic cultures}

The organotypic cultures were produced using the Gravity Plus system (InSphero, Zurich, Switzerland). According to the manufacturer's instructions, $40 \mu \mathrm{l}$ of cell suspension was given into each well of the plate for the formation of multi-cellular spheroids (250 - 8000 cells). For initial seeding, Williams Medium E supplemented with $10 \%$ FCS was used. It was changed by refreshing $50 \%$ of the culture volume every 2-3 days. For the quantification of metabolites, the supernatants were collected, centrifuged and stored at $-20^{\circ} \mathrm{C}$ until analysis. The spheroids were monitored using a 10x long-working distance objective of an Olympus IX 70 microscope which was connected to an Olympus CC12 Soft Imaging System (Muenster, Germany).

\section{Maintenance in low-serum conditions}

The spheroids were produced using medium supplemented with $10 \%$ FCS. After spheroid formation, 50\% medium volume was replaced three times. This time point was set as day 1 in low-serum conditions. However, due to the fact that the medium cannot be replaced completely in the drop, a small amount of $1.25 \%$ FCS was still present in the medium. After that, medium was replaced at day 4 reducing the percentage of serum to below $1 \%$. Medium drops without cells were used as controls for each time point.

\section{AST assay}

The activity of liver-specific aspartate aminotransferase (AST) in the culture supernatant as cell viability marker was determined using a kinetic UV assay kit (Hitado, Moehnesee- Delecke, Germany) according to the manufacturer's instructions. A dilution series of standard serum (NobiCal-Multi, Hitado) was measured in parallel for quantification.

\section{Quantification of glucose, albumin and amino acids}

D-glucose and L-lactate concentrations in the supernatants were determined using routinely utilized enzymatic kits (R-Biopharm, Darmstadt, Germany). The assays were performed according to the instructions of the manufacturer.

Albumin concentration was determined by an enzyme-linked immunosorbent assay (ELISA) (Albuwell II; Exocell, Philadelphia, USA) according to manufacturer's instructions.

Concentrations of amino acids in the supernatants were quantified by an high performance liquid chromatography (HPLC) method as previously reported [18].

Medium exchange was performed by refreshing $50 \%$ of the drop volume. The metabolic rates in $3 \mathrm{D}$ spheroid cultures were determined using the following equation:

$$
r=\left[\left(0.5 \times c_{m}+0.5 \times c_{d x-1}\right)-c_{d x}\right] \times V / \Delta t
$$

Whereas

$\mathrm{c}_{\mathrm{m}}=\mathrm{c}$ (metabolite) in medium w/o cells

$c_{\mathrm{dx}}=\mathrm{c}$ (metabolite) in supernatant at day $\mathrm{x}$

$c_{\mathrm{dx}-1}=\mathrm{c}$ (metabolite) in supernatant at day $\mathrm{x}-1$

$\mathrm{V}=$ hanging drop volume

\section{Live/dead-assay}

Live/dead-assay was performed using fluoresceindiacetate/ propidiumiodide staining (FDA/PI). Cells were incubated with FDA/ PI staining solution ( $25 \mu \mathrm{M}$ FDA / $40 \mu \mathrm{M}$ PI in PBS) for $1 \mathrm{~min}$. After PBS washing $(2 \mathrm{x})$, fluorescence was monitored using an Olympus IX70 fluorescence microscope (ex $488 \mathrm{~nm}$ ).

\section{CYP450 induction assay}

For the induction of CYP1A, the HepG2 cells were incubated with $5 \mu \mathrm{M}$ 3-methylcholantren (3-MC) for $72 \mathrm{~h}$. EROD assay, based on the conversion of 7-ethoxyresorufin to the fluorescent dye resorufin by CYP1A, was performed. For that, the cells were incubated with the substrate 7 -ethoxyresorufin $(10 \mu \mathrm{M}$ in serum-free medium) for 
3 h. Fluorescence at ex/em 544/590 nm was measured. Fluorescence intensities were corrected for background fluorescence (substrate solution without cells). Fold changes compared to uninduced control were calculated.

\section{Dose response curves}

To assess dose-dependent toxic effects on the cells in the three different cultivation types, stock solution of tamoxifen was prepared in DMSO. Concentrations of $0.1-500 \mu \mathrm{M}$ were tested in triplicates. For all experiments, the highest DMSO concentration did not exceed $2 \%$. Cells were incubated with the respective drug concentrations in serumfree medium for $24 \mathrm{~h}$. After $24 \mathrm{~h}$ drug exposure, alamar blue assay was performed. For this, alamar blue assay solution $(20 \% \mathrm{v} / \mathrm{v})$ was added to the cells and incubated for $4 \mathrm{~h}$. Fluorescence was measured using a Fluoroskan Ascent CF fluorescence reader (Thermo Labsystems, Vantaa, Finland) at ex/em 544/590 nm. Three individual experiments were carried out for each cultivation system and mean values of viability related to the respective untreated control were calculated. Dose-response curves were obtained by plotting the logs of the tested concentrations against the viability as percentage of untreated control. $\mathrm{EC}_{50}$ values and standard deviations were determined using the Boltzmann function (Origin 8.1G).

\section{MRP-2 transporter activity}

A fluorescence based assay was used for the investigation of MRP2 transporter activity in organotypic cultures. Thereby, the membrane permeable and non-fluorescent substrate 5-chloromethylfluorescein diacetate (CMFDA) was used as substrate. CMFDA is converted by cellular esterases to a membrane-impermeable compound, which then reacts with cellular glutathione to glutathione-methylfluorescein (GSMF). GSMF is a substrate of the membrane transporter MRP-2 and is excreted out of the cell into canaliculi where it accumulates. The organotypic cultures were incubated at day 3 after seeding with $5 \mu \mathrm{M}$ CMFDA for $30 \mathrm{~min}$. Thereafter, dye solution was aspirated and the cells were incubated with dye-free medium for $45 \mathrm{~min}$. Fluorescence was monitored using an Olympus IX 70 fluorescence microscope (Muenster, Germany).

\section{Statistical analysis}

The fold induction in organotypic, monolayer and collagen sandwich cultures relative to respective uninduced controls were compared using student's $t$-test (Matlab R2006a). The $\mathrm{EC}_{50}$ values in OTC, ML and CS cultures were compared using student's $t$-test (Matlab R2006a).

\section{Results}

\section{Spheroid formation and growth}

Spheroid formation and growth was investigated by seeding different cell numbers $(250$ - 8000). Morphology was evaluated over seven days as shown in figure 1. Structure reorganization was observed already at day 1 after seeding. Compact organotypic cultures were observed at day 2-3. The used cell numbers all formed compact spheroids of reproducible diameters. Moreover, cell proliferation was observed by increasing cluster sizes. In the group of 250 seeded cells at day 1 , diameters of $500 \mu \mathrm{m}$ were achieved at day 7 . In case of 8000 initial seeded cells, the spheroid diameters were around $900 \mu \mathrm{m}$ at day 7. It was also apparent that the spheroids could freely move in the drop.

Organotypic cultures of 2000 seeded cells were further analyzed regarding time-dependent growth by measuring the diameters over 10 days (Figure 2). After initial seeding, the diameter decreased due to cell reorganization and tissue-like formation. The spheroids were then growing linearly $\left(\mathrm{R}^{2}=0.98\right)$ to a diameter of $630 \pm 11 \mu \mathrm{m}$ at day 6 . After that, the cluster size then decreased until day 10 to $592 \pm 21 \mu \mathrm{m}$. For further experiments, 2000 cells were chosen as initial cell number which gives sufficient signal in viability assay and can be cultivated with high viability for more than two weeks.

\section{Maintenance of the spheroids in low-serum conditions}

After structure reorganization, the HepG2 spheroids were maintained in very low serum medium $(<1 \%)$. For viability, AST release from HepG2 spheroids was measured (Figure 3a). The highest AST activity $(0.67 \mathrm{mU} / \mathrm{d})$ was found after adjusting low serum conditions (day 3 ) and was constant until day 6 indicating highest cell death in this phase. Thereafter, AST release significantly decreased to stable values

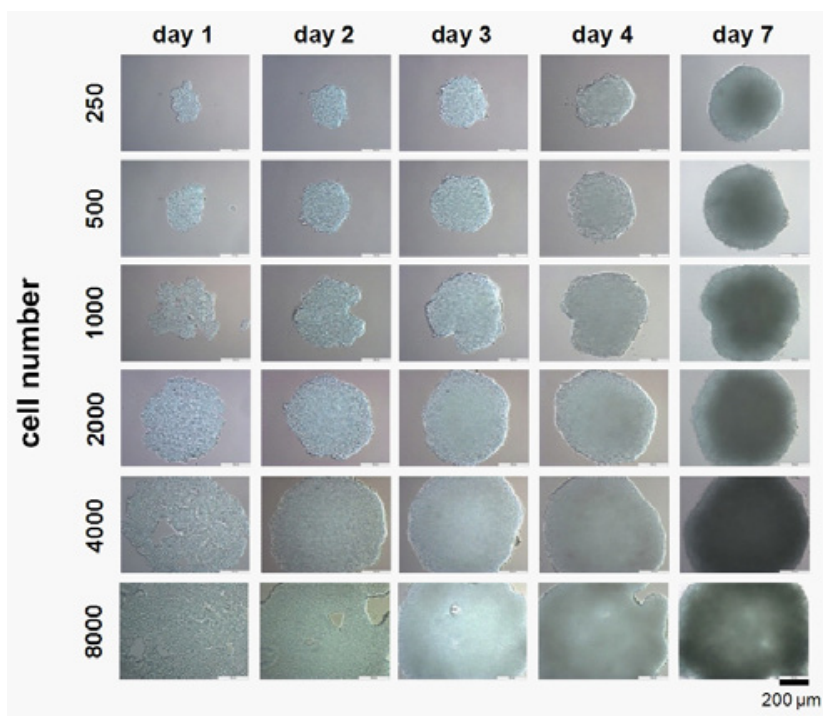

Figure 1: Formation of HepG2 spheroids. Different cell numbers $(250-8000)$ were seeded and cluster formation was monitored over seven days as indicated. Scale bars represent $200 \mu \mathrm{m}$.

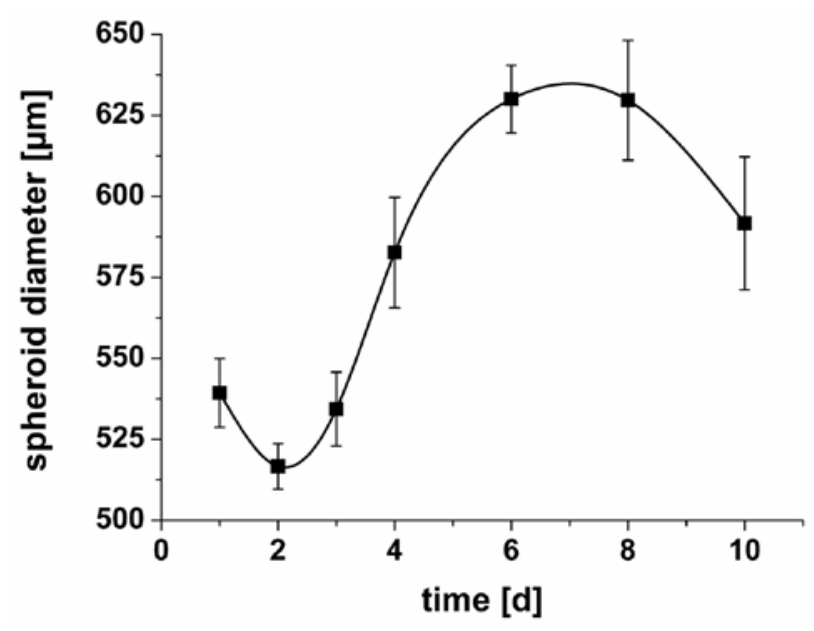

Figure 2: Spheroid growth over time. 2000 cells were seeded (day 0 ) and the diameter was measured until day 10 . Error bars represent $\pm \operatorname{SD}(n=3)$. 
a
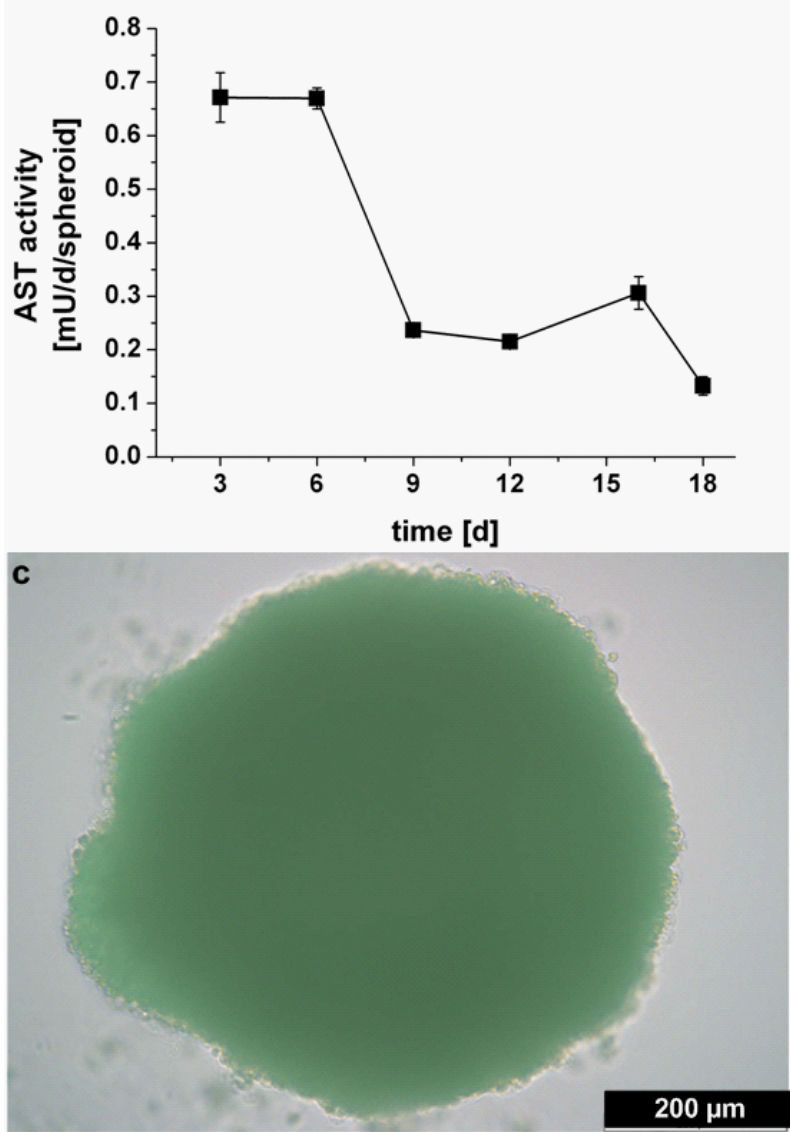

b
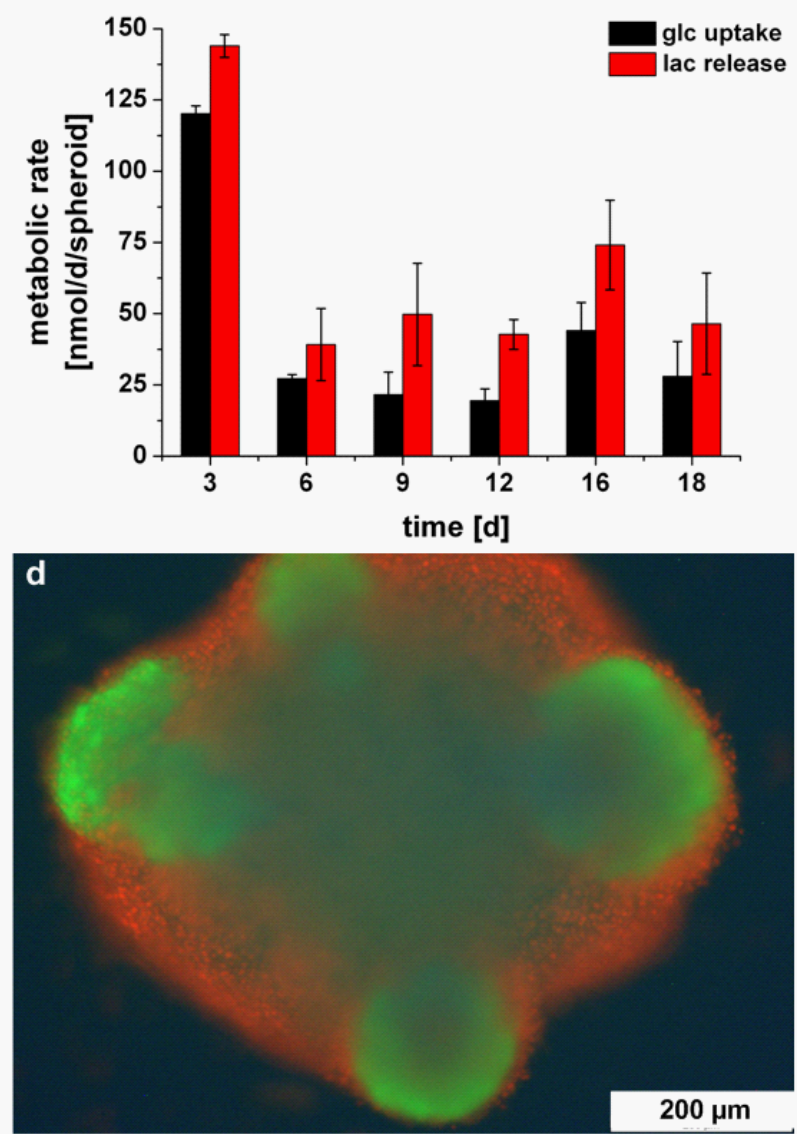

Figure 3: Low-serum, long-term cultivation of HepG2 cells in organotypic cultures a) AST activity b) glucose consumption and lactate production rates c) morphology of organotypic culture at day $18 \mathrm{~d}$ ) viability of organotypic culture at day 18 , assessed by Live-Dead staining. Error bars in a) and b) indicate $\pm \mathrm{SD}$ ( $n=3$, supernatants of 6 spheroids were pooled). Day $1=1^{\text {st }}$ day in low-serum medium. Scale bars represent $200 \mu \mathrm{m}$.

in the middle phase of cultivation. At day 18, the lowest AST activity $(0.13 \mathrm{mU} / \mathrm{d})$ was found in the spheroid supernatants.

The spheroids consumed glucose with highest rate at day 3 (120 $\mathrm{nmol} / \mathrm{d}$ ). This rate decreased during low-serum maintenance to nearly constant values between day 4 and 18. Similarly, the highest lactate production (144 nmol/d) was measured at day 3 after adjusting serumfree conditions which decreased further on to values between 39 and $74 \mathrm{nmol} / \mathrm{d}$. Lactate yield was highest between days 9-12, whereas at the beginning and at the end of the cultivation, lactate yields between 1.2-1.7 were observed.

Light microscopy revealed that the organotypic cultures still maintained their 3D structure at day 16 (Figure 3c). Though high cell death rate was observed by live/dead staining (Figure $3 \mathrm{~d}$ ), some outer parts of the spheroids showed high viability indicating high proliferation rates in these zones.

Albumin production in HepG2 cells in the three cultivation systems was measured (Figure 4). It was observed that the organotypic cultures produced the highest amounts of albumin (normalized to the initial seeded cell number). The albumin production per seeded cell increased in both monolayer- and collagen sandwich cultures over 16 days indicating cell proliferation. The organotypic cultures produced the highest amount of albumin at day 3 (38 pg/d/seeded cell). Between day
6 and day 16 in low-serum medium, the production rates were almost constant $(8-13 \mathrm{pg} / \mathrm{d} / \mathrm{seeded}$ cell). Comparing the three cultivation systems, the albumin production rates were 17 or 38 fold higher in the organotypic cultures than in monolayer- or collagen sandwich cultures at day 3. At day 16 , fold changes the production rates per seeded cell were more similar (fold changes 1.4 for ML and 1.6 for CS).

Amino acid metabolism in the HepG2 spheroids in low-serum medium was analyzed as shown in figure 5 . At day 6 , glutamine (3.7 $\mathrm{nmol} / \mathrm{d})$, alanine $(2.8 \mathrm{nmol} / \mathrm{d})$, leucine $(1.2 \mathrm{nmol} / \mathrm{d})$ and arginine (1.1 $\mathrm{nmol} / \mathrm{d}$ ) were consumed with highest rates. The spheroids produced high amounts of glutamate between day 6 and $18(2.8-4.5 \mathrm{nmol} / \mathrm{d})$. The consumption of serine increased after the first cultivation week as well as aspartate production decreased over time. For glycine and proline, we found low net production rates in the later phase. The branchedchain amino acids leucine, isoleucine and valine were constantly taken up until day 18. Glutamine was consumed over the whole cultivation at very stable rates $(3.6-4.3 \mathrm{nmol} / \mathrm{d})$

\section{CYP450 induction}

We analyzed the CYP1A induction capacity of HepG2 cells in monolayer, collagen-sandwich and organotypic cultures using the EROD assay. The organotypic cultures clearly showed the highest fold change (44.6) upon 3-MC induction compared to the uninduced 


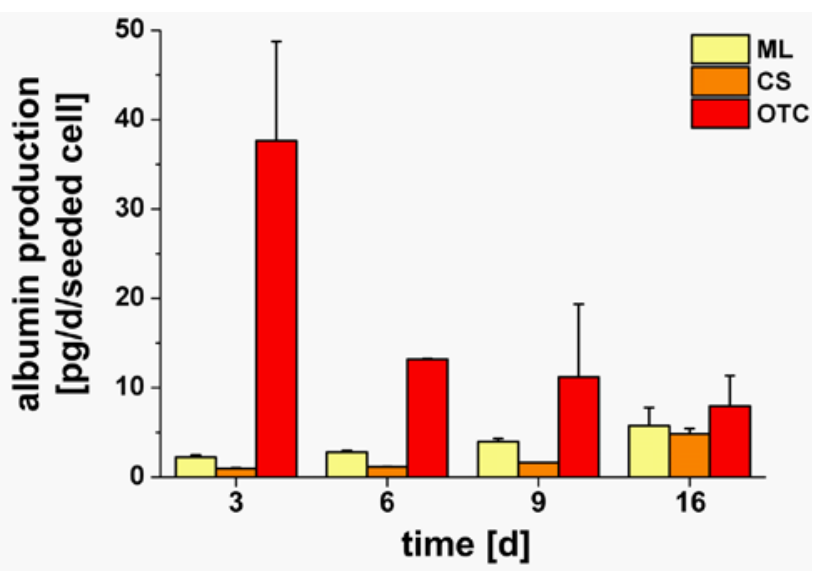

Figure 4: Albumin production rates in monolayer (ML), collagen sandwich (CS) and organotypic (OTC) cultures, expressed in $\mathrm{pg} / \mathrm{d} / \mathrm{seeded}$ cell. Error bars indicate standard deviation $(n=2$, for OTC supernatants of 6 spheroids were pooled).

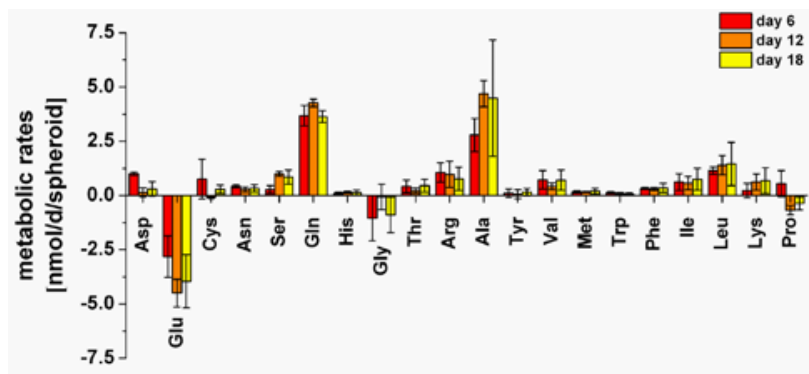

Figure 5: Amino acid metabolism in 3D spheroids during low-serum long term cultivation at day 6,12 and 18 respectively. Positive vales indicate net uptake. Day $1=1$ st day in serum-free conditions. Error bars indicate $\pm S D$ $(n=3$, supernatants of 6 spheroids were pooled).

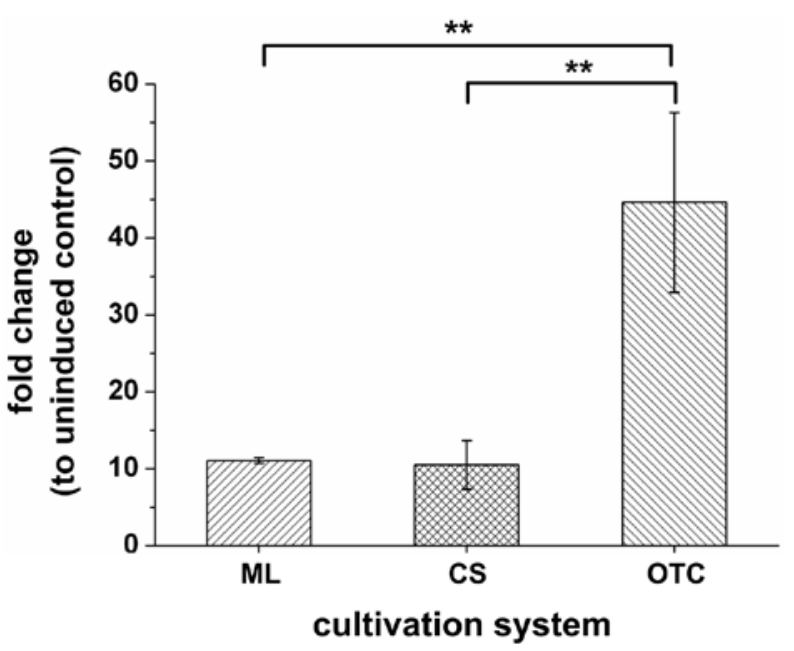

Figure 6: CYP1A induction assessed by EROD assay. Induction fold change (relative to uninduced control) after $72 \mathrm{~h}$ induction with 3-MC is shown for HepG2 cells cultivated in monolayer (ML), collagen-sandwich (CS) or organotypic cultures (OTC). Error bars indicate $\pm S D(n=3)$. ${ }^{* *}$ Significance at $p<0.01$. control (Figure 6). The induction capacity was significantly higher compared to the other two cultivation systems $(p<0.01)$. Monolayerand collagen-sandwich-cultures showed similar induction capacities (fold changes 10.6 and 10.5, respectively).

\section{Toxicity of tamoxifen}

The toxicity of tamoxifen was assessed in monolayer-, collagen sandwich- and organotypic cultures. Dose-dependent effects of the drug were observed for all three cell culture systems (Figure 7 a-c). The $\mathrm{EC}_{50}$ value (Figure $7 \mathrm{~d}$ ) for monolayer cultures $(13.9 \mu \mathrm{M})$ was significantly lower as compared to collagen-sandwich culture (19.1 $\mu \mathrm{M})$. The organotypic cultures showed the highest $\mathrm{EC}_{50}$ value $(56.8 \mu \mathrm{M})$ of the three models. The effects of tamoxifen on the HepG2 spheroids were further visualized by live-dead-staining. Untreated control, 50 $\mu \mathrm{M}$ (in the range of $\mathrm{EC}_{50}$ ) and $100 \mu \mathrm{M}$ as positive control were tested (incubation time $24 \mathrm{~h}$ ) as depicted in figure 7. The untreated spheroids (Figure $8 \mathrm{a}$, d) were highly compact and showed only a low number of dead cells around the cluster. After exposure to $50 \mu \mathrm{M}$ tamoxifen, a higher amount of dead cells were observed in the drop (Figure $8 \mathrm{~b}$ ) as well as on the cluster surface (Figure $8 \mathrm{e}$ ). Cell debris as shown by light microscopy in figure $8 \mathrm{~b}$ was washed away during the staining procedure. The treatment with $100 \mu \mathrm{M}$ tamoxifen degraded the spheroid structure and its compactness and induced cell death; both at the surface and within the organotypic culture (Figure $8 \mathrm{c}$ and $\mathrm{f}$ ).

\section{Investigation of MRP-2 activity}

The MRP-2 transporter activity was investigated using CMFDAbased assay. Monolayer cultures of HepG2 show strong intracellular fluorescence (Figure 9a) and almost no transport of the dye into canaliculi. Likewise high accumulation of the fluorescent (Figure 9b) dye within the cells was found for the collagen sandwich cultures. In contrast, the organotypic cultures show MRP-2 transporter activity all around the 3D structure. The fluorescent MRP-2 substrate was transported out of the cell. Accumulation within the canaliculi between cells was clearly observed whereas no intracellular fluorescence was detected (Figure 9d).

\section{Discussion}

In this study, 3D organotypic cultivation of HepG2 cells was performed in a scaffold-free system using hanging drop method. The method was already applied for the formation of organotypic cultures of different cell types and cell lines [34-37]. Tissue reorganization is facilitated by gravity and as shown in our study, organotypic cultures (OTC) of a wide range of initial cells numbers $(250-8000)$ can be produced. Scaffold-free cellular reorganization was observed already at day 1 after seeding and was completed at day 3 . The scaffold-free system enables the microtissue formation without any force besides gravity and without xenogenic or synthetic materials. Therefore, biodegradability is not an issue and the produced organotypic cultures can be used as alternative to scaffold-based microtissue building blocks.

Importantly, the sizes of microtissues are reproducible $(< \pm 10 \%$ of mean) and are exactly adjustable for each experiment. In case of toxicity studies, lower cell numbers e.g. $250-500$ could be used for long-term, chronic experiments ( $>3$ weeks) in case of proliferating cells and higher numbers $(2000-8000)$ could be applied in short-term toxicity studies for which a certain cell number is needed to perform endpoint assays. The low cell numbers required for these organotypic spheroids can moreover enhance throughput by allowing parallel studies in multiwell plates. This is especially advantageous in case of 
a
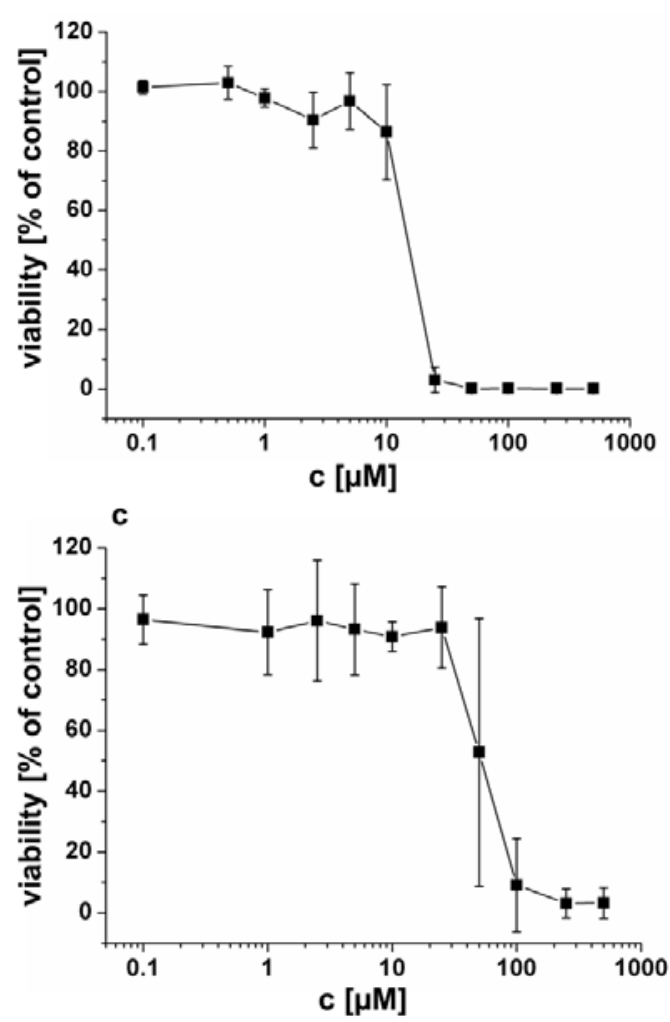

b
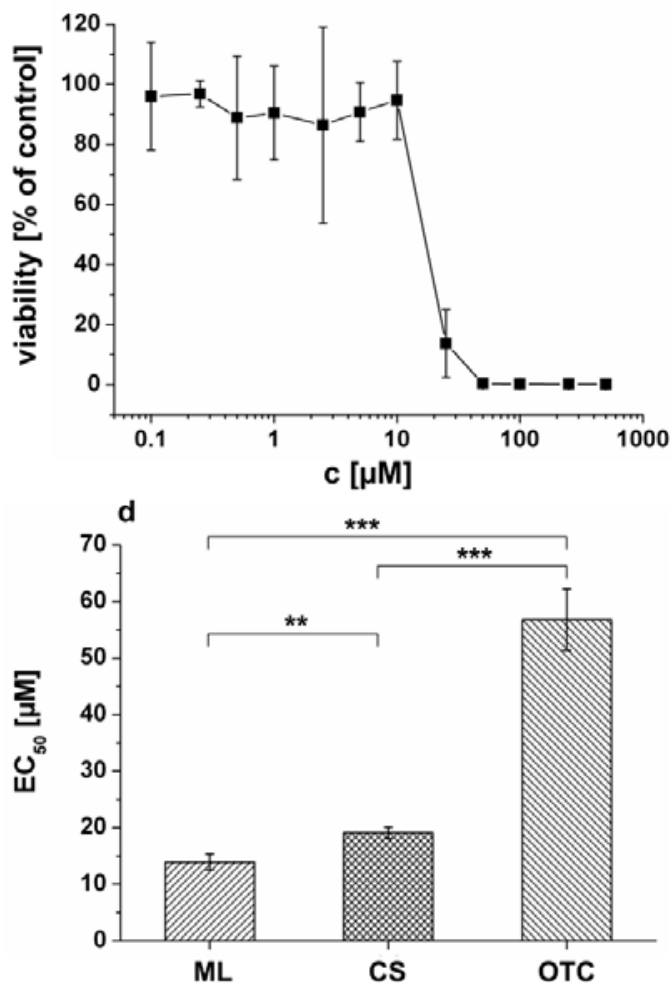

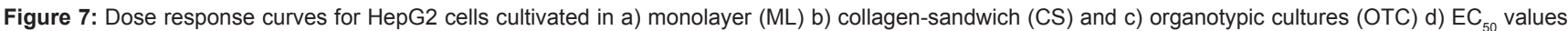
of tamoxifen ( $24 \mathrm{~h}$ exposure). $\mathrm{EC}_{50}$ values were calculated using Boltzmann function. Viability was assessed by alamar blue assay and calculated relative to the respective untreated controls. Error bars indicate $\pm \mathrm{SD}(\mathrm{N}=3, n=3),{ }^{* *}$ significance at $p<0.01$, ${ }^{* *}$ significance at $p<0.001$.

a
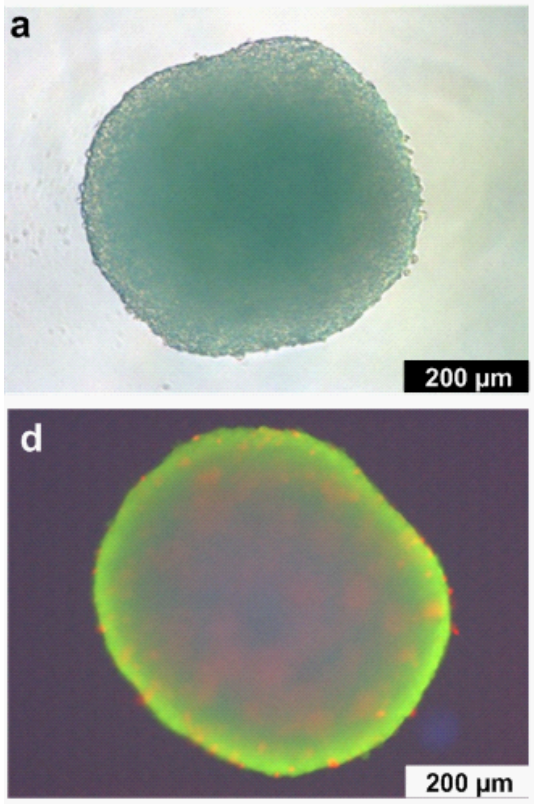

b
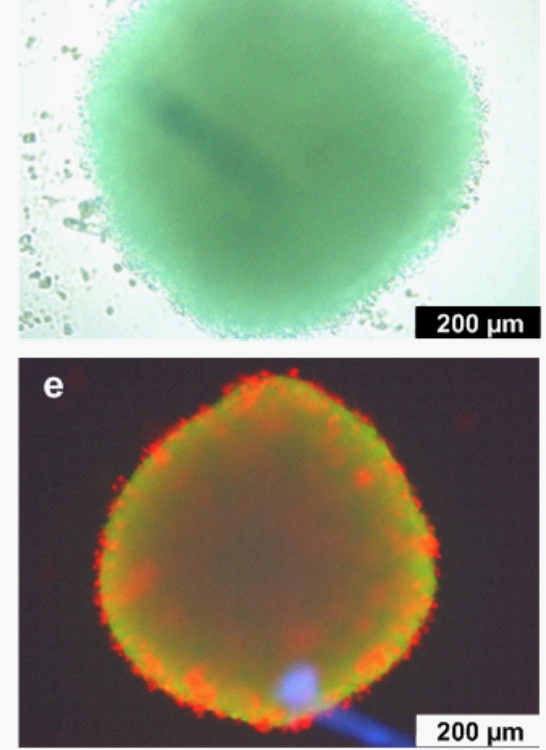

C
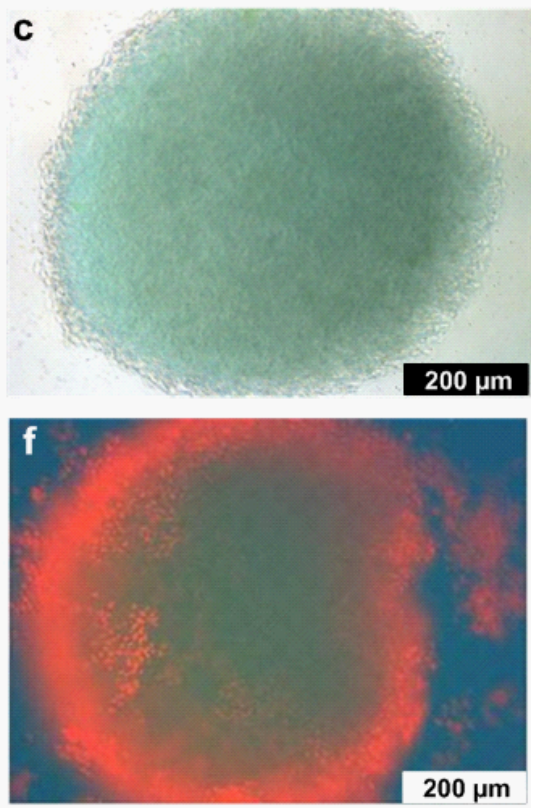

Figure 8: Morphology and viability of HepG2 organotypic cultures after $24 \mathrm{~h}$ exposure to tamoxifen. Morphology: a) untreated control b) $50 \mu \mathrm{M}$ tamoxifen c) $100 \mu \mathrm{M}$ tamoxifen; Live/dead staining (FDA/PI) in d) untreated control e) $50 \mu \mathrm{M}$ tamoxifen f) $100 \mu \mathrm{M}$ tamoxifen. Scale bars represent $200 \mu \mathrm{m}$. 
Citation: Mueller D, Koetemann A, Noor F (2011) Organotypic Cultures of Hepg2 Cells for In Vitro Toxicity Studies. J Bioengineer \& Biomedical Sci S2:002. doi:10.4172/2155-9538.S2-002

primary human cells which are limited by availability and other costly cell types. In case of hepatotoxicity, adequate replicates from same donor can be included. The change of culture medium is possible and therefore, different conditions are adjustable in the system e.g. the reduction of serum, the exposure to drugs, induction and other activity studies as shown in our study. Supernatant collection moreover allows studies such as metabolite profiling or protein analysis. Therefore, a detailed analysis of single spheroids is possible. If needed, high numbers of spheroids can rapidly be generated, harvested and used for further analysis. We maintained the HepG2 spheroids in low-serum medium since reduction of serum is desirable due to its chemically undefined composition, batch to batch variations and possible interactions of serum components with drug or inducers. The spheroids could be kept viable for more than two weeks at very low serum $(<1 \%)$ conditions. In the late cultivation phase, proliferation zones within the spheroids were detected. This was also confirmed by glucose uptake and lactate production rates, which increased at the end of the cultivation (day 16) probably due to cell proliferation. The activity of AST as marker of cell death was highest directly after adjusting to low-serum medium. This might be due to high cellular stress in this phase because of several
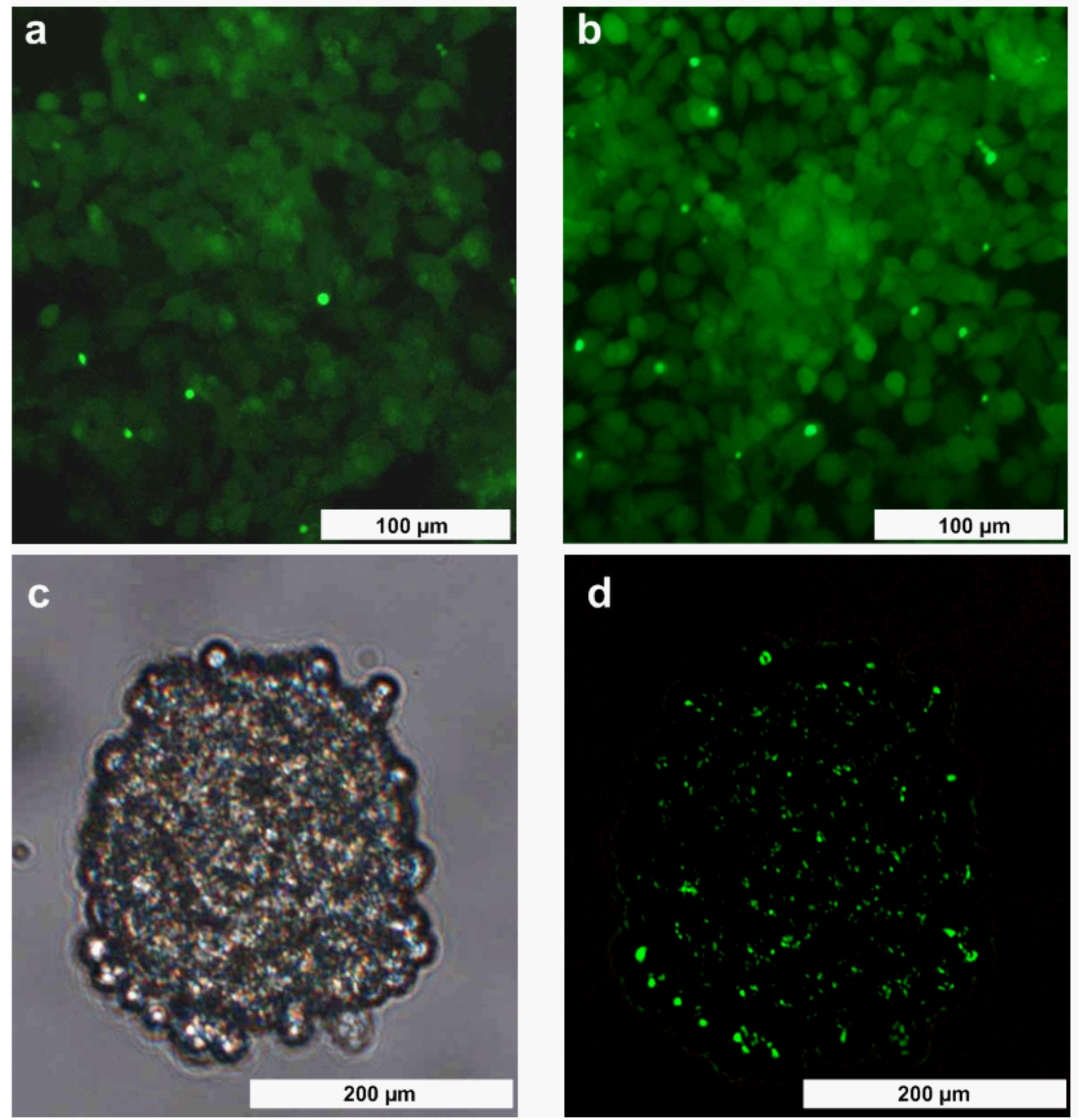

Figure 9: CMFDA-based fluorescence assay for MRP-2 transporter activity a) HepG2 monolayer culture (CY2 filter, fluorescence microscopy) b) HepG2 sandwich culture (CY2 filter, fluorescence microscopy) c) HepG2 organotypic culture (light microscopy) d) HepG2 organotypic culture (CY2 filter, fluorescence microscopy). 
medium change steps. Between day $3-6$, AST release was at constant high level indicating constant number of dying cells during this time. However, growing size indicated proliferation of the cells at the same time which seemed to stop after day 6 and onwards. Thus, AST activity was at a low stable level between day 6 and 16. Albumin production rates in the organotypic cultures were highest $(38 \mathrm{pg} / \mathrm{d} / \mathrm{seeded}$ cell) at day 3 in low-serum medium (representing day 6 after seeding), as seen before for glucose and lactate uptake/production rates. This rate is quite close to in vivo values of $48-72 \mathrm{pg} / \mathrm{d} /$ hepatocyte [38]. Albumin production in OTC was almost stable between days 6 and 16 and higher than in monolayer (ML) and collagen sandwich (CS) cultures for each time point. As observed by diameter measurements, the spheroids $(2000$ seeded cells) stopped increasing in size upon achieving diameters of about $625 \mu \mathrm{m}$. This is probably due to contact inhibition which usually does not occur in conventional 2D cultures [39]. It was reported that three-dimensionally grown HepG2 cultures show higher cell cycle arrest and therefore lower proliferation than monolayer cultures [20]. This may explain stable albumin production between day 6 and 16 by OTC since HepG2 cells seems to have stopped proliferating. Whereas in case of ML and CS the cells are continuously proliferating and therefore the albumin production per seeded cell number is higher.

Nevertheless, the increased serum protein production show enhanced liver-specific function of the organotypic cultures. The analysis of amino acid metabolism reveals that the spheroids consumed most of the proteinogenic amino acids over the whole low-serum cultivation of 3 weeks. The highest rates were found for glutamine, acting as the main energy source for the spheroids besides glucose. Moreover, mammalian cells use glutamine nitrogen to build nucleotides, amino acids and vitamins. On the other side, the spheroids steadily produced glutamate which plays an important role in the degradation and transamination of various amino acids. BCAAs were constantly consumed over time as additional energy source. Theses amino acids can be converted to acetyl-CoA or succinyl-CoA, which subsequently enter the TCA cycle. BCAAs moreover induce albumin production in hepatocytes through the mTOR signal pathway [40]. Overall, the consumption and production rates for most of the amino acids were quite constant indicating stable metabolism and viability of the spheroids which is a prerequisite for chronic toxicity assessment. Therefore, these organotypic cultures are well-suited for investigations of long-term drug effects. Moreover, assessing the metabolic profile of the organotypic cultures would help define substrates for ${ }^{13} \mathrm{C}$ metabolic flux analysis (MFA). This can further give deep insights into the cellular metabolism of the organotypic cultures and contribute to the analysis of drug-induced metabolic effects. The use of MFA in physiological characterization has been recently reviewed [41-43]. Additionally, analyzing the influence of the initial cell number (250-8000) on cellular metabolism would be of high interest for future studies, since differences in nutrient supply, diffusion through the spheroid, oxygen concentration or $\mathrm{pH}$ could affect the metabolic rates in the organotypic cultures. Further studies in these directions are underway in our laboratory.

The assessment and prediction of CYP450 enzyme induction by xenobiotics is one of the main tasks in early drug development [44]. We tested the CYP1A induction capacity, as the predominant CYP450 isoform in HepG2 cells [30], and compared organotypic cultures to monolayer and collagen sandwich cultures. Enzyme induction was highest in the OTC and induction in ML and CS culture was similar at lower level. CYP1A enzymes catalyze the oxygenation of polycyclic aromatic hydrocarbons (PAHs) and heterocyclic aromatic amines / amides (HAAs) which can result in the formation of carcinogens [45].
Therefore, CYP1A induction is still of main interest in cancer research but also in drug development [46]. The induction of CYP1A by a certain substrate can result in an enhanced metabolism of another substance. In drug development, the assessment of such drug-drug interactions is important, emphasizing the need of in vitro cell culture systems with functional CYP inducibility. The HepG2 organotypic cultures respond better to the CYP1A inducer 3-MC than conventional cultures and are therefore applicable as in vitro model for testing CYP1A induction by drug candidates.

The short-term toxicity ( $24 \mathrm{~h}$ exposure) of tamoxifen was tested in the three culture systems. It was reported that tamoxifen causes cytotoxicity in tumor cell lines [33] and human hepatocytes [47]. For HepG2 cells, it was shown that tamoxifen down regulates the expression of survivin gene thereby inhibits proliferation [48]. Concentrationdependent toxic effects were assessed in our study for HepG2 cells in each of the three cultivation systems. The $\mathrm{EC}_{50}$ value assessed for conventional monolayer culture was similar to a recently reported value [49]. For the HepG2 cells cultivated in collagen-sandwich culture, we assessed a higher $\mathrm{EC}_{50}$ value showing indicating an influence of the extracellular matrix. However, the HepG2 spheroids clearly showed the highest $\mathrm{EC}_{50}$ value compared with the other two culture systems. This means that the cells in the organotypic cultures are less sensitive to the anti-cancer drug tamoxifen. Tamoxifen and its metabolites are substrates of ATP-binding cassette (ABC) superfamily members such as MDR-1 or MRP-2 [50]. It has been reported that HepG2 spheroids show higher MDR-1 activity compared to monolayer cultures [29]. In our study, we show that MRP-2 activity is also significantly higher in the organotypic cultures compared to conventional cultures of HepG2. This could probably lead to an increased efflux of tamoxifen. Moreover, tamoxifen itself induces the expression of MDR-1 and MRP-2 [51,52] which furthermore contribute to the lower sensitivity of the spheroids to this drug.

The monitoring of morphology and viability showed that mainly the cells on the spheroid surface were affected by exposure to a tamoxifen concentration in the range of the EC50 value $(50 \mu \mathrm{M})$. This is probably due to the first contact of the drug to the outer cells. However, by exposure to a higher drug concentration $(100 \mu \mathrm{M})$, it was clearly shown that also the inner parts of the organotypic cultures were impaired indicating complete diffusion of the drug through the spheroids. The transport of extracellular factors, signal molecules, or drugs through the liver spheroids as well as the formation of gap junctions or bile canaliculi is being further investigated in detail in our lab.

We show that spheroids of different sizes could be produced and maintained viable for more than two weeks in low serum $(<1 \%)$ conditions. The 3D tissue like structure enhanced liver-specific functions such as CYP450 enzyme induction. The assessment of tamoxifen toxicity clearly revealed higher $\mathrm{EC}_{50}$ values for the $3 \mathrm{D}$ cultures indicating that the spheroids are less sensitive to this anticancer drug. Moreover, we could show the formation of bile canaliculi in the organotypic cultures as well as the increased expression of MRP2 membrane transporter compared to conventional cultures.

\section{Conclusion}

We report the engineering of HepG2 organotypic cultures with adjustable sizes. The cells reorganize spontaneously and built organotypic cultures (OTC) in the hanging drop without any physical force except gravity. They were characterized including growth, longterm maintenance in low-serum medium, cellular metabolism and CYP450 induction. The toxic potential of tamoxifen was assessed 
Citation: Mueller D, Koetemann A, Noor F (2011) Organotypic Cultures of Hepg2 Cells for In Vitro Toxicity Studies. J Bioengineer \& Biomedical Sci S2:002. doi:10.4172/2155-9538.S2-002

and the results were compared to monolayer and collagen-sandwich cultures. We show that the spheroid cultivation system could be applied to long-term studies. The system has several advantages since both supernatant as well as spheroid sampling is possible, culture conditions can be changed easily, reproducible spheroid sizes are achieved and it is well-suited for high throughput experiments. The micro-tissues achieved represent a more in vivo like situation and can be used as in vitro model using cell lines to screen anti-cancer drugs and to study tumor-specific mechanisms, e.g. chemotherapy resistance. Primary human cells can also be used for long term studies specially in preclinical drug development.

\section{Acknowledgments}

We acknowledge the excellent technical assistance of Esther Hoffmann and Michel Fritz.

\section{References}

1. Cukierman E, Pankov R, Stevens DR, Yamada KM (2001) Taking cell-matrix adhesions to the third dimension. Science 294: 1708-1712.

2. Cukierman E, Pankov R, Yamada KM (2002) Cell interactions with threedimensional matrices. Curr Opin Cell Biol 14: 633-639.

3. Tibbitt MW, Anseth KS (2009) Hydrogels as extracellular matrix mimics for 3D cell culture. Biotechnol Bioeng 103: 655-663.

4. Godoy P, Hengstler JG, Ilkavets I, Meyer C, Bachmann A, et al. (2009) Extracellular matrix modulates sensitivity of hepatocytes to fibroblastoid dedifferentiation and transforming growth factor beta-induced apoptosis. Hepatology 49: 2031-2043.

5. Mikos AG, Bao Y, Cima LG, Ingber DE, Vacanti JP, et al. (1993) Preparation of poly(glycolic acid) bonded fiber structures for cell attachment and transplantation. J Biomed Mater Res 27: 183-189.

6. Temenoff, J. S., and Mikos, A. G. (2000) Review: tissue engineering for regeneration of articular cartilage. Biomaterials 21: 431-440

7. Fisher JP, Jo S, Mikos AG, Reddi AH (2004) Thermoreversible hydroge scaffolds for articular cartilage engineering. J Biomed Mater Res A 71: 268-274.

8. Liebmann T, Rydholm S, Akpe V, Brismar H (2007) Self-assembling Fmoc dipeptide hydrogel for in situ 3D cell culturing. BMC Biotechnol 7: 88 .

9. Park SH, Park SR, Chung SI, Pai KS, Min BH (2005) Tissue-engineered cartilage using fibrin/hyaluronan composite gel and its in vivo implantation. Artif Organs 29: 838-845.

10. Shim JH, Kim JY, Park M, Park J, Cho DW (2011) Development of a hybrid scaffold with synthetic biomaterials and hydrogel using solid freeform fabrication technology. Biofabrication 3: 034102.

11. Miranda JP, Rodrigues A, TostÃues RM, Leite S, Zimmerman H, et al. (2010) Extending hepatocyte functionality for drug-testing applications using highviscosity alginate-encapsulated three-dimensional cultures in bioreactors. Tissue Eng Part C Methods 16: 1223-1232.

12. TostÃues RM, Leite SB, Miranda JP, Sousa M, Wang DI, et al. (2011) Perfusion of 3D encapsulated hepatocytes--a synergistic effect enhancing long-term functionality in bioreactors. Biotechnol Bioeng 108: 41-49.

13. Frisk T, Rydholm S, Liebmann T, Svahn HA, Stemme G, et al. (2007) A microfluidic device for parallel 3-D cell cultures in asymmetric environments. Electrophoresis 28: 4705-4712.

14. Gerlach JC, Mutig K, Sauer IM, Schrade P, Efimova E (2003) Use of primary human liver cells originating from discarded grafts in a bioreactor for liver support therapy and the prospects of culturing adult liver stem cells in bioreactors: a morphologic study. Transplantation 76: 781-786.

15. Sauer IM, Neuhaus P, Gerlach JC (2002) Concept for modular extracorporeal liver support for the treatment of acute hepatic failure. Metab Brain Dis 17: 477-484.

16. van de Kerkhove MP, Di Florio E, Scuderi V, Mancini A, Belli A, et al. (2002) Phase I clinical trial with the AMC-bioartificial liver. Int J Artif Organs 25: 950 959
17. van de Kerkhove MP, Di Florio E, Scuderi V, Mancini A, Belli A, et al. (2003) Bridging a patient with acute liver failure to liver transplantation by the AMCbioartificial liver. Cell Transplant 12: 563-568.

18. Mueller D, Tascher G, Müller-Vieira U, Knobeloch D, Nuessler AK, et al. (2011) In-depth physiological characterization of primary human hepatocytes in a 3D hollow-fiber bioreactor. J Tissue Eng Regen Med 5: e207-218.

19. Miranda JP, Leite SB, Muller-Vieira U, Rodrigues A, Carrondo MJ, et al. (2009) Towards an extended functional hepatocyte in vitro culture. Tissue Eng Part $C$ Methods 15: 157-167.

20. Li CL, Tian T, Nan KJ, Zhao N, Guo YH, et al. (2008) Survival advantages of multicellular spheroids vs. monolayers of HepG2 cells in vitro. Oncol Rep 20 1465-1471.

21. Mandenius CF, Andersson TB, Alves PM, Batzl-Hartmann C, Björquist $P$ et al. (2011) Toward preclinical predictive drug testing for metabolism and hepatotoxicity by using in vitro models derived from human embryonic stem cells and human cell lines - a report on the Vitrocellomics EU-project. Altern Lab Anim 39: 147-171.

22. Beckers S, Noor F, Müller-Vieira U, Mayer M, Strigun A, et al. (2010) High throughput, non-invasive and dynamic toxicity screening on adherent cells using respiratory measurements. Toxicol In Vitro 24: 686-694.

23. Niklas J, Noor F, Heinzle E (2009) Effects of drugs in subtoxic concentrations on the metabolic fluxes in human hepatoma cell line Hep G2. Toxicol Appl Pharmacol 240: 327-336

24. Noor F, Niklas J, Müller-Vieira U, Heinzle E (2009) An integrated approach to improved toxicity prediction for the safety assessment during preclinical drug development using Hep G2 cells. Toxicol Appl Pharmacol 237: 221-231.

25. Bazou D, Coakley WT, Hayes AJ, Jackson SK (2008) Long-term viability and proliferation of alginate-encapsulated 3-D HepG2 aggregates formed in an ultrasound trap. Toxicol In Vitro 22: 1321-1331.

26. Corstorphine L, Sefton MV (2011) Effectiveness factor and diffusion limitation in collagen gel modules containing HepG2 cells. J Tissue Eng Regen Med 5 : 119-129.

27. Lang R, Stern MM, Smith L, Liu Y, Bharadwaj S, et al. (2011) Three-dimensiona culture of hepatocytes on porcine liver tissue-derived extracellular matrix. Biomaterials 32: 7042-7052.

28. Nakamura K, Mizutani R, Sanbe A, Enosawa S, Kasahara M, et al. (2011) Evaluation of drug toxicity with hepatocytes cultured in a micro-space cel culture system. J Biosci Bioeng 111: 78-84.

29. Oshikata A, Matsushita T, Ueoka R (2011) Enhancement of drug efflux activity via MDR1 protein by spheroid culture of human hepatic cancer cells. J Biosci Bioeng 111: 590-593.

30. Wilkening S, Stahl F, Bader A (2003) Comparison of primary human hepatocytes and hepatoma cell line Hepg2 with regard to their biotransformation properties. Drug Metab Dispos 31: 1035-1042.

31. Bhadriraju K, Chen CS (2002) Engineering cellular microenvironments to improve cell-based drug testing. Drug Discov Today 7: 612-620.

32. Holmes FA, Liticker JD (2005) Pharmacogenomics of tamoxifen in a nutshelland who broke the nutcracker? J Oncol Pract 1: 155-159.

33. Petinari L, Kohn LK, de Carvalho JE, Genari SC (2004) Cytotoxicity of tamoxifen in normal and tumoral cell lines and its ability to induce cellular transformation in vitro. Cell Biol Int 28: 531-539.

34. Kelm JM, Ehler E, Nielsen LK, Schlatter S, Perriard JC, et al. (2004) Design of artificial myocardial microtissues. Tissue Eng 10: 201-214.

35. Kelm JM, Fussenegger M (2004) Microscale tissue engineering using gravityenforced cell assembly. Trends Biotechnol 22: 195-202.

36. Kelm JM, Timmins NE, Brown CJ, Fussenegger M, Nielsen LK (2003) Method for generation of homogeneous multicellular tumor spheroids applicable to a wide variety of cell types. Biotechnol Bioeng 83: 173-180.

37. Kelm JM, Ittner LM, Born W, Djonov V, Fussenegger M (2006) Self-assembly of sensory neurons into ganglia-like microtissues. J Biotechnol 121: 86-101.

38. Bhatia SN, Balis UJ, Yarmush ML, Toner M (1999) Effect of cell-cel interactions in preservation of cellular phenotype: cocultivation of hepatocytes and nonparenchymal cells. FASEB J 13: 1883-1900. 
Citation: Mueller D, Koetemann A, Noor F (2011) Organotypic Cultures of Hepg2 Cells for In Vitro Toxicity Studies. J Bioengineer \& Biomedical Sci S2:002. doi:10.4172/2155-9538.S2-002

39. Maruyama M, Matsunaga T, Harada E, Ohmori S (2007) Comparison of basal gene expression and induction of CYP3As in HepG2 and human fetal liver cells. Biol Pharm Bull 30: 2091-2097.

40. ljichi C, Matsumura T, Tsuji T, Eto $Y$ (2003) Branched-chain amino acids promote albumin synthesis in rat primary hepatocytes through the mTOR signal transduction system. Biochem Biophys Res Commun 303: 59-64.

41. Niklas J, Heinzle E (2011) Metabolic Flux Analysis in Systems Biology of Mammalian Cells. Adv Biochem Eng Biotechnol.

42. Niklas J, Sandig V, Heinzle E (2011) Metabolite channeling and compartmentation in the human cell line AGE1.HN determined by (13)C labeling experiments and (13)C metabolic flux analysis. J Biosci Bioeng

43. Niklas J, Schneider K, Heinzle E (2011) Metabolic flux analysis in eukaryotes. Curr Opin Biotechnol 21: 63-69.

44. LeCluyse EL (2001) Human hepatocyte culture systems for the in vitro evaluation of cytochrome P450 expression and regulation. Eur J Pharm Sci 13: $343-368$

45. Jerina DM (1983) The 1982 Bernard B. brodie Award Lecture. Metabolism of Aromatic hydrocarbons by the cytochrome P-450 system and epoxide hydrolase. Drug Metab Dispos 11: 1-4.

46. Ma Q, Lu AY (2007) CYP1A induction and human risk assessment: an evolving tale of in vitro and in vivo studies. Drug Metab Dispos 35: 1009-1016.
47. Li AP, Bode C, Sakai Y (2004) A novel in vitro system, the integrated discrete multiple organ cell culture (IdMOC) system, for the evaluation of human drug toxicity: comparative cytotoxicity of tamoxifen towards normal human cells from five major organs and MCF-7 adenocarcinoma breast cancer cells. Chem Bio Interact 150: 129-136.

48. Guo R, Huang Z, Shu Y, Jin S, Ge H (2009) Tamoxifen inhibits proliferation and induces apoptosis in human hepatocellular carcinoma cell line HepG2 via down-regulation of survivin expression. Biomed Pharmacother 63: 375-379.

49. Gerets HH, Hanon E, Cornet M, Dhalluin S, Depelchin O, et al. (2009) Selection of cytotoxicity markers for the screening of new chemical entities in a pharmaceutical context: a preliminary study using a multiplexing approach. Toxicol In Vitro 23: 319-332.

50. Shin SC, Choi JS, Li X (2006) Enhanced bioavailability of tamoxifen after ora administration of tamoxifen with quercetin in rats. Int J Pharm 313: 144-149.

51. Nagaoka R, Iwasaki T, Rokutanda N, Takeshita A, Koibuchi Y, et al. (2006) Tamoxifen activates CYP3A4 and MDR1 genes through steroid and xenobiotic receptor in breast cancer cells. Endocrine 30: 261-268.

52. Kauffmann HM, Keppler D, Gant TW, Schrenk D (1998) Induction of hepatic mrp2 (cmrp/cmoat) gene expression in nonhuman primates treated with rifampicin or tamoxifen. Arch Toxicol 72: 763-768. 